\title{
Gender and Racial Disparities in Initial Hemodialysis Access and Outcomes in Incident End-Stage Renal Disease Patients
}

\author{
Silvi Shah ${ }^{\mathrm{a}}$ Anthony C. Leonard ${ }^{\mathrm{b}}$ Karthikeyan Meganathan ${ }^{\mathrm{c}}$ \\ Annette L. Christianson ${ }^{c}$ Charuhas V. Thakara, d \\ ${ }^{a}$ Division of Nephrology Kidney C.A.R.E. Program, University of Cincinnati, Cincinnati, OH, USA; ${ }^{b}$ Department of Family \\ and Community Medicine, University of Cincinnati, Cincinnati, $\mathrm{OH}, \mathrm{USA}$; 'Department of Biomedical Informatics, \\ University of Cincinnati, Cincinnati, $\mathrm{OH}, \mathrm{USA} ;{ }^{\mathrm{d}}$ Cincinnati VA Medical Center, Cincinnati, $\mathrm{OH}$, USA
}

\section{Keywords}

Disparities · Gender · Race · Vascular access ·

Hemodialysis · Mortality

\begin{abstract}
Background: Arteriovenous (AV) access confers survival benefits over central venous catheters (CVC) in hemodialysis patients. Although chronic kidney disease disproportionately affects women and racial minorities, disparities in the utilization of hemodialysis access across Asians, Native Americans, Hispanics, blacks, and whites among males and females after accounting for pre-dialysis health are not well studied. Methods: We evaluated 885,699 patients with end-stage renal disease who initiated hemodialysis between January 1, 2004 and December 31, 2014 using the US Renal Data System. Multivariable logistic regression models adjusted for pre-dialysis health were used to test the associations between gender and race on type of vascular access (AV access vs. CVC, and AV fistula vs. AV graft) at hemo-
\end{abstract}

dialysis initiation as primary outcome, and on 1-year mortality as a secondary outcome. Results: Mean age was $65 \pm$ 14 years. Females were less likely to use AV access for hemodialysis initiation than were males $(\mathrm{OR} 0.85 ; 95 \% \mathrm{Cl} 0.84$ 0.86). Compared to whites, adjusted odds of AV access for hemodialysis initiation were higher in blacks (OR 1.08; 95\% Cl 1.07-1.70), Asians (OR 1.11; 95\% Cl 1.07-1.14); and lower in Hispanics (OR 0.89; $95 \% \mathrm{Cl} 0.87-0.90)$. There was no significant difference in mortality between males and females. Compared to whites, 1-year adjusted mortality was lower in Asians (OR 0.55; 95\% Cl 0.53-0.56), blacks (OR 0.67; $95 \% \mathrm{Cl} 0.66-0.68$ ), Hispanics (OR 0.62; 95\% Cl 0.61-0.63), and Native Americans (OR 0.62; 95\% Cl 0.58-0.66). Conclusion: Females had lower odds of using AV access than do males for hemodialysis initiation. As compared to whites, blacks and Asians were more likely, and Hispanics were less likely to use AV access for first outpatient hemodialysis. Further investigation of biological and process of care factors may help in developing ways to reduce these disparities.

(c) 2018 The Author(s)

Published by S. Karger AG, Basel

\begin{tabular}{ll}
\hline KARGER & $\begin{array}{l}\text { Ko 2018 The Author(s) } \\
\text { Published by S. Karger AG, Basel }\end{array}$ \\
E-Mail karger@karger & Opecess \\
www.karger.com/ajn & This article is licensed under the Creative Commons Attribution- \\
& $\begin{array}{l}\text { NonCommercial-NoDerivatives 4.0 International License (CC BY- } \\
\text { NC-ND) (http://www.karger.com/Services/OpenAccessLicense). } \\
\text { Usage and distribution for commercial purposes as well as any dis- } \\
\text { tribution of modified material requires written permission. }\end{array}$
\end{tabular}

Silvi Shah, MD, FASN, FACP

Assistant Professor of Medicine

Division of Nephrology, University of Cincinnati

231 Albert Sabin Way, MSB 6112, Cincinnati, OH 45267 (USA)

E-Mail shah2sv@ucmail.uc.edu 


\section{Introduction}

End-stage renal disease (ESRD) is a global public health problem that affects more than 660,000 people in United States, and is characterized by high morbidity and mortality [1]. ESRD is notable for poorly understood differences in its prevalence or complications across gender and race $[2,3]$. While the prevalence of early stages of chronic kidney disease is similar across different races, ESRD disproportionately affects the nation's minorities. For example, compared to whites, the prevalence of ESRD is 3.7 times greater in blacks, 1.4 times greater in Native Americans, and 1.5 higher in Asians and Hispanics [4-6]. Even though Asians are the fastest growing racial group of the United States population, outcomes in Asian patients with ESRD are not well studied [7]. Hence, research on potential disparities in the provision of health care during the pre-ESRD period and its associated clinical outcomes in ESRD patients become relevant.

The type of vascular access at the initiation of hemodialysis remains a key outcome that impacts survival as well as resource utilization among patients with ESRD. The clinical practice guidelines for vascular access recommend the use of arteriovenous (AV) accesses - native fistulae or synthetic grafts over central venous catheters (CVC) for hemodialysis, due to lower risk of mortality and morbidity $[8,9]$. Furthermore, CVC results in high resource utilization due to the costs associated with procedural complications, infections, and hospitalizations $[10,11]$. Differences in biological factors associated with gender and race can influence the type of vascular access at incident dialysis. Women are shown to have lower probability of receiving AV fistula than men [12]. Zarkowsky et al. [13] reported that Hispanic or black patients tend to use AV fistula less frequently than white patients to initiate hemodialysis; but did not consider AV graft as one of the parameters of successful AV access [13]. Prior studies stratified by race did not look at gender differences among them, or took into account Asian and Native American patients. Additionally, there are no studies that have comprehensively accounted for all of the available factors determining the pre-dialysis health status (for example, functional status, pre-ESRD nephrology care, comorbidities, employment status, and laboratory parameters of anemia and low albumin) that could potentially be associated with the type of vascular access in patients with ESRD [14].

By utilizing information from US Renal Data System (USRDS), and accounting for differences in pre-dialysis health, we evaluated the type and frequency of hemodialysis access use in incident ESRD patients across males and females in 5 different racial categories. One of our objectives was to examine the impact of gender differences between white vs. black vs. Hispanic vs. Asians vs. Native Americans ESRD population on incident vascular access.

\section{Material and Methods}

\section{Study Population and Data Sources}

An observational cohort study of a national database was performed using 885,699 adult patients with ESRD who initiated hemodialysis between January 1, 2004, and December 31, 2014 from the USRDS. We identified all patients in the USRDS with a first ESRD service date for maintenance hemodialysis from January 1, 2004, to December 31, 2014 who had not received a kidney transplant $(n=1,106,937)$. We excluded patients who were on peritoneal dialysis or who had missing information on dialysis modality $(n=108,142)$, or on vascular access $(n=111,324)$, race $(n=1,707)$, or gender $(n=65)$. Patients were followed for mortality from dialysis initiation to the end of the study period, August 1, 2016 giving each patient at least 12 months of follow-up. The University of Cincinnati review boards approved this study and waived the need for informed consent. Figure 1 demonstrates the study cohort derivation.

A physician is required to certify the Centers for Medicare and Medicaid Services (CMS) medical evidence report form-2728 for incident hemodialysis patients. We used information derived from form CMS-2728 to determine demographics (age, race, gender, body mass index), hemodialysis modality, vascular access (AV fistula, AV graft, catheter, and others), comorbidities (congestive heart failure, atherosclerotic heart disease including other cardiac disease, hypertension, diabetes mellitus, cancer, peripheral vascular disease, stroke, chronic obstructive pulmonary disease, and amputation), employment history, insurance, pre-dialysis nephrology care, nursing home residence, cause of ESRD, laboratory data (serum albumin and serum hemoglobin), functional status, and access to transplant information. Dates of death were obtained from USRDS patients' file. We included the following covariates to determine the pre-dialysis health in patients with ESRD: pre-dialysis nephrology care, nursing home residence, functional status, comorbidities, and laboratory data (serum hemoglobin and serum albumin). The primary cause of death was ascertained from form CMS-2746 (cardiovascular disease, infections, malignancy, withdrawal of dialysis and others/unknown) [2].

\section{Outcome and Predictors}

The primary outcome of this study was the type of vascular access at the time of initiation of hemodialysis and modeled as (1) AV access (which included AV fistula and AV graft) versus CVC (which included catheter and others); and (2) AV fistula versus AV graft. The secondary outcome was 1-year mortality. Gender was categorized as male and female. Race was grouped into Hispanic, non-Hispanic white, non-Hispanic black, non-Hispanic Asian, and non-Hispanic Native American. 


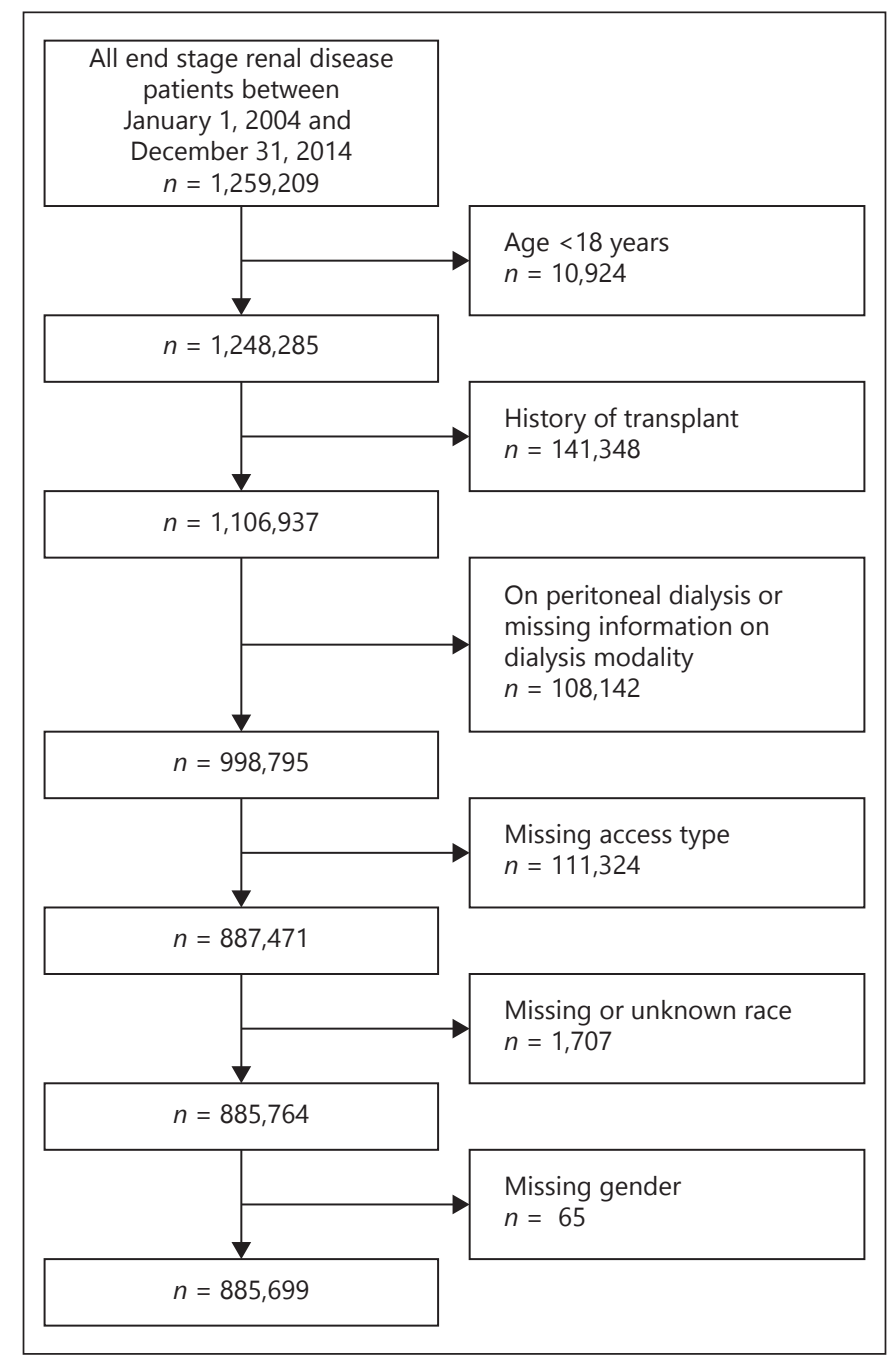

Fig. 1. Flow diagram showing the cohort selection process.

\section{Statistical Analyses}

Bivariate relationships between diagnostic categories and comorbid diseases, on the one hand, and race and gender, on the other hand, were tested with the chi-square test or Fisher's exact test for categorical variables, or $t$ tests or one-way ANOVA for continuous variables. Summary statistics were presented as percentages for categorical variables and mean \pm SD for continuous variables. We examined associations between race and gender and the type of vascular access (AV access versus CVC, and AV fistula versus AV graft) in hemodialysis patients, using multivariable logistic models. Explanatory variables in the non-parsimonious logistic regression models included gender, race, octogenarian status, body mass index, diabetes mellitus, hypertension, congestive heart failure, atherosclerotic heart disease, chronic obstructive pulmonary disease, peripheral vascular disease, amputation, serum albumin, serum hemoglobin, cause of ESRD, nursing home residence, access to transplant information, and pre-ESRD nephrology care. Comparative risk estimates were expressed as OR and 95\% CI. Statistical significance was set at a 2 -tailed $p$ value of 0.05 , unadjusted for multiple tests. All data were analyzed using SAS version 9.4 (SAS Institute, Cary, NC, USA).

\section{Results}

The mean age of the patients was $65 \pm 14$. The majority, $56.3 \%$, was male. Most of the patients were white (53.2\%), followed by black (28.1\%), Hispanic (13.7\%), Asian (4.1\%), and Native American (0.9\%). Overall, CVC was the dominant hemodialysis access type, used in $82.5 \%$ of the patients. Table 1 shows the baseline characteristics in the entire study cohort and for males and females. Females had higher rates of obesity ( 43.5 vs. $33.6 \%, p<$ 0.001 ), diabetes ( 59.3 vs. $55.1 \%, p<0.001$ ), and congestive heart failure ( 35.7 vs. $34.2 \%, p<0.001)$ than did male patients. Additionally, females were younger and were more frequently employed and insured. Females had higher rates of poor functional status ( 18.6 vs. $14.5 \%, p<0.001$ ) and were more likely to have a history of nursing home residence ( 9.7 vs. $7.4 \%, p<0.001$ ), than were males. Low albumin $<3.5$ (51.8 vs. $50.1 \%, p<0.001)$ and low hemoglobin $<11$ ( 72.4 vs. $69.3 \%, p<0.001)$ were more frequent in females. Female patients had a slightly lower frequency of $\mathrm{AV}$ access for hemodialysis initiation than male patients ( 16.2 vs. $18.5 \%, p<0.001$ ). Unadjusted 1 -year mortality was similar for males and females.

Table 2 shows the baseline characteristics across different races for the incident ESRD population. Whites had the highest rate of congestive heart failure (38.2\%), whereas Native Americans had the highest rate of diabetes (78.0\%). Hispanics, blacks, and Native Americans were more likely to receive no pre-dialysis nephrology care followed by whites and Asians (35.2, 33, 32.4, 28.9, and $27.6 \%$ respectively, $p<0.001$ ). With regards to octogenarian status, whites had the highest rate, while Native Americans had the lowest rate (22.0 vs. $5.2 \%)$. In unadjusted analyses, Asians had the highest frequency of AV access use followed by whites, whereas Hispanics had the lowest frequency of $\mathrm{AV}$ access for hemodialysis initiation $(20,18$, and $14.6 \%$ respectively).

Figure 2 shows the distribution of types of vascular access across gender and race in the incident hemodialysis population. Among females, the frequency of AV access was highest in Asians followed by blacks, Native Americans, whites, and Hispanics (18.7, 16.7, 16.7, 16.4, and $13.6 \%)$. Among males, the frequency of AV access was highest in Asians followed by whites, blacks, Native Americans, and Hispanics (21.1, 19.2, 18.3, 17.9, and 
Table 1. Patient characteristics associated with gender

\begin{tabular}{|c|c|c|c|c|}
\hline Characteristics & $\begin{array}{l}\text { Total } \\
(n=885,699)\end{array}$ & $\begin{array}{l}\text { Male } \\
(n=498,339 ; 56.3 \%)\end{array}$ & $\begin{array}{l}\text { Female } \\
(n=387,360 ; 43.7 \%)\end{array}$ & $p$ value \\
\hline \multicolumn{5}{|l|}{ Demographics } \\
\hline Age, years & $64(14)$ & $64(14)$ & $65(14)$ & $<0.001$ \\
\hline Octogenarians & 16.4 & 15.9 & 17.0 & $<0.001$ \\
\hline BMI $(n=877,105), \mathrm{kg} / \mathrm{m}^{2}$ & $29.3(8.2)$ & $28.6(7.4)$ & $30.3(9.0)$ & $<0.001$ \\
\hline $25-29.99$ & 27.6 & 30.2 & 24.2 & \\
\hline$\geq 30$ & 37.9 & 33.6 & 43.5 & \\
\hline Missing & 1.0 & 1.0 & 1.0 & \\
\hline Race & & & & $<0.001$ \\
\hline Asian & 4.1 & 4.0 & 4.1 & \\
\hline Black & 28.1 & 25.7 & 31.1 & \\
\hline \multicolumn{5}{|l|}{ Comorbidities } \\
\hline Congestive heart failure & 34.9 & 34.2 & 35.7 & $<0.001$ \\
\hline ASHD & 34.9 & 36.9 & 32.4 & $<0.001$ \\
\hline Hypertension & 85.7 & 85.1 & 86.4 & $<0.001$ \\
\hline Diabetes mellitus & 57.0 & 55.1 & 59.3 & $<0.001$ \\
\hline Cancer & 8.1 & 8.9 & 7.0 & $<0.001$ \\
\hline Amputation & 3.5 & 4.2 & 2.6 & $<0.001$ \\
\hline PVD & 14.4 & 15.5 & 13.0 & $<0.001$ \\
\hline TIA/CVA & 10.1 & 9.9 & 10.4 & $<0.001$ \\
\hline COPD & 10.5 & 10.6 & 10.4 & 0.006 \\
\hline \multicolumn{5}{|l|}{ Hemodialysis access } \\
\hline $\mathrm{AV}$ access & 17.5 & 18.5 & 16.2 & $<0.001$ \\
\hline$<3.5$ & 50.8 & 50.1 & 51.8 & $<0.001$ \\
\hline$\geq 3.5$ & 23.2 & 24.1 & 21.9 & \\
\hline Missing & 26.0 & 25.8 & 26.2 & \\
\hline Hemoglobin $(n=707,818), \mathrm{g} / \mathrm{dL}$ & $9.8(1.8)$ & $9.9(1.8)$ & $9.7(1.7)$ & $<0.001$ \\
\hline$<11$ & 70.7 & 69.3 & 72.4 & $<0.001$ \\
\hline $11-12$ & 11.9 & 12.2 & 11.4 & \\
\hline$>12$ & 7.5 & 8.5 & 6.3 & \\
\hline Missing & 9.9 & 9.9 & 9.9 & \\
\hline \multicolumn{5}{|l|}{ Dialysis setting } \\
\hline Dialysis facility & 99.0 & 99.0 & 99.0 & $<0.001$ \\
\hline Home & 0.3 & 0.3 & 0.3 & \\
\hline Skilled nursing facility & 0.7 & 0.6 & 0.8 & \\
\hline \multicolumn{5}{|l|}{ Employment status } \\
\hline Unemployed & 21.7 & 20.7 & 23.1 & $<0.001$ \\
\hline Employed + others & 13.2 & 11.8 & 15.0 & \\
\hline Retired-age & 42.9 & 44.3 & 41.2 & \\
\hline Retired-disability & 22.2 & 23.3 & 20.7 & \\
\hline
\end{tabular}


Table 1. (continued)

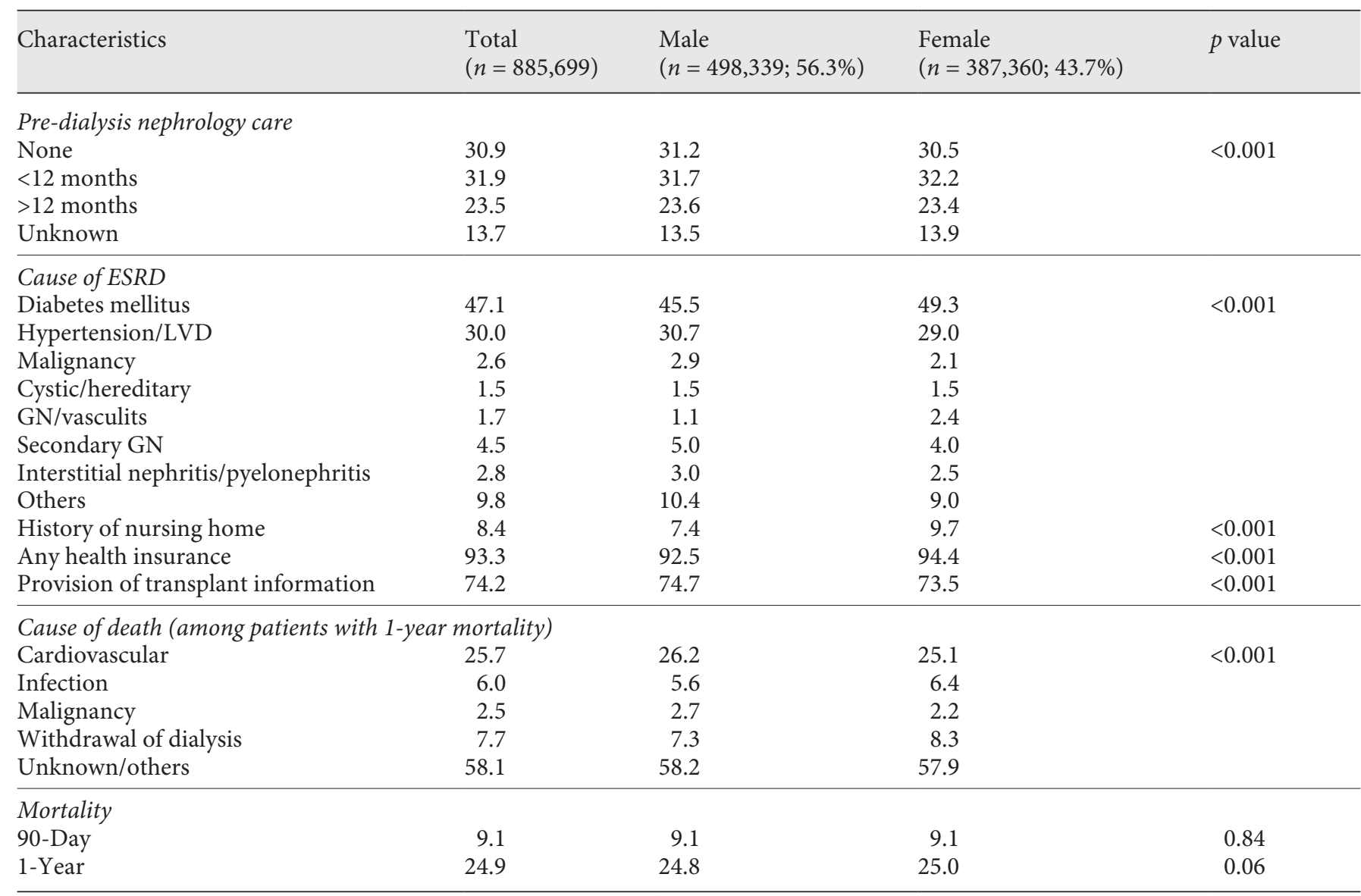

Data are presented in mean (SD), or proportion where appropriate. BMI, body mass index; ASHD, atherosclerotic heart disease; CVA/TIA, cerebrovascular accident/transient ischemic attack; COPD, chronic obstructive pulmonary disease; PVD, peripheral vascular disease; AV, arteriovenous; CVC, central venous catheter; LVD, large vessel disease; GN, glomerulonephritis.

15.4\%). Overall, Asian males had the highest rates of AV access, whereas Hispanic males had the lowest rates of AV access (21.1 vs. $15.4 \%)$. With regards to the use of AV graft for hemodialysis initiation, the highest rates were noted in black females, and lowest rates were present in Native American males (5.8 vs. 1.6\%). There was no significant interaction between race and gender for the outcome of AV access vs. CVC ( $p=0.69)$, or AV fistula vs. AV graft $(p=0.10)$ for the initiation of hemodialysis in incident ESRD patients.

Figure 3a shows the unadjusted and adjusted odds ratios across gender and across the 5 races predicting hemodialysis initiation with an AV access vs. CVC. Females had lower adjusted odds of using AV access for hemodialysis initiation (OR 0.85; 95\% CI 0.84-0.86; $p<$ 0.001 ). Black patients had higher adjusted odds of initiating hemodialysis with $\mathrm{AV}$ access as compared to white patients (OR 1.08; 95\% CI 1.07-1.10; $p<0.001$ ). Asians were also more likely to use AV access for initiation of hemodialysis than were whites (OR 1.11; 95\% CI 1.07$1.14 ; p<0.001)$. In contrast, Hispanics had lower odds of using AV access to start hemodialysis in incident ESRD patients as compared to whites (OR 0.89; 95\% CI 0.87$0.90, p<0.001)$. The odds of AV access versus CVC were not statistically different in Native American patients compared to that of white patients (OR 1.04; 95\% CI $0.98-1.10)$.

Within the AV access type, we modeled the incident use of AV fistula compared to AV graft. Figure $3 \mathrm{~b}$ shows the unadjusted and adjusted odds ratios across gender and across 5 different races predicting the initiation of hemodialysis with an AV fistula versus AV graft. Females were less likely to use AV fistula over AV graft than were males (OR 0.86; 95\% CI 0.87-0.90, $p<0.001$ ). As com- 
Table 2. Patient characteristics associated with race

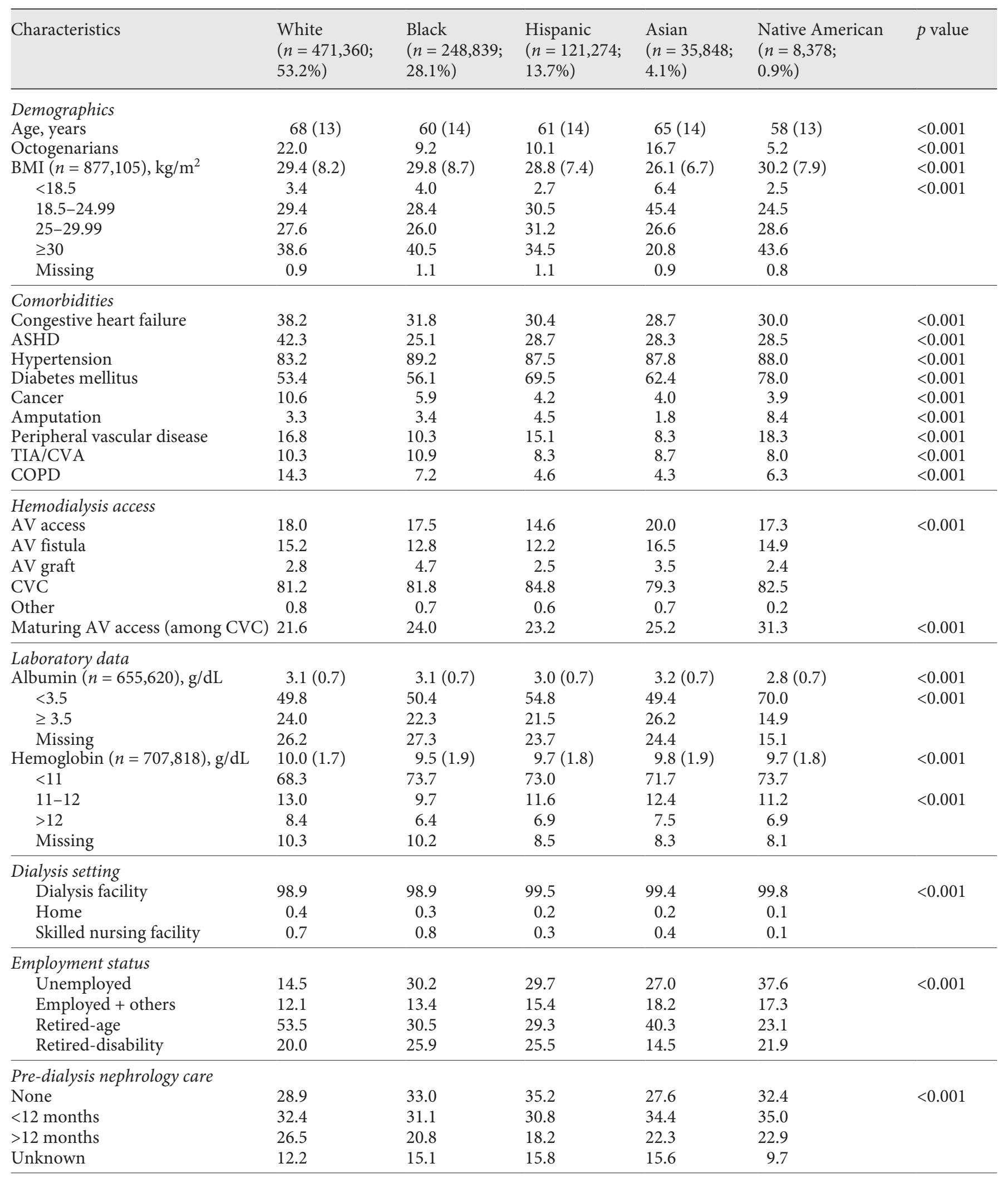


Table 2. (continued)

\begin{tabular}{|c|c|c|c|c|c|c|}
\hline Characteristics & $\begin{array}{l}\text { White } \\
(n=471,360 ; \\
53.2 \%)\end{array}$ & $\begin{array}{l}\text { Black } \\
(n=248,839 ; \\
28.1 \%)\end{array}$ & $\begin{array}{l}\text { Hispanic } \\
(n=121,274 ; \\
13.7 \%)\end{array}$ & $\begin{array}{l}\text { Asian } \\
(n=35,848 \\
4.1 \%)\end{array}$ & $\begin{array}{l}\text { Native American } \\
(n=8,378 \\
0.9 \%)\end{array}$ & $p$ value \\
\hline $\mathrm{DM}$ & 42.8 & 44.6 & 64.6 & 56.3 & 73.8 & \multirow[t]{7}{*}{$<0.001$} \\
\hline HTN/LVD & 29.1 & 37.7 & 19.8 & 25.5 & 10.5 & \\
\hline Malignancy & 3.5 & 1.6 & 1.4 & 1.2 & 1.1 & \\
\hline GN/vasculits & 1.7 & 1.8 & 1.5 & 1.4 & 1.7 & \\
\hline Secondary GN & 4.9 & 4.0 & 3.8 & 6.4 & 4.7 & \\
\hline Interstitial nephritis/pyelonephritis & 3.9 & 1.3 & 1.6 & 2.0 & 1.5 & \\
\hline Others & 12.1 & 7.9 & 6.2 & 6.1 & 5.9 & \\
\hline History of nursing home & 9.8 & 7.9 & 5.0 & 5.0 & 4.7 & \multirow[t]{2}{*}{$<0.001$} \\
\hline Any health insurance & 96.1 & 90.1 & 89.4 & 93.2 & 92.9 & \\
\hline Malignancy & 2.9 & 2.2 & 1.7 & 1.8 & 1.5 & \multirow{3}{*}{$<0.001$} \\
\hline Withdrawal of dialysis & 11.0 & 3.7 & 4.5 & 4.2 & 6.3 & \\
\hline Unknown/others & 52.5 & 65.2 & 63.4 & 63.3 & 63.8 & \\
\hline \multicolumn{7}{|l|}{ Mortality } \\
\hline 90-Day & 11.6 & 6.4 & 6.1 & 5.8 & 5.3 & $<0.001$ \\
\hline 1-Year & 30.6 & 19.0 & 17.8 & 16.8 & 16.5 & $<0.001$ \\
\hline
\end{tabular}

Data are presented in mean (SD), or proportion where appropriate. BMI, body mass index; ASHD, atherosclerotic heart disease; CVA/TIA, cerebrovascular accident/transient ischemic attack; COPD, chronic obstructive pulmonary disease; AV, arteriovenous; CVC, central venous catheter; LVD, large vessel disease; GN, glomerulonephritis.

pared to whites, Native Americans had higher odds of using AV fistula for hemodialysis initiation (OR 1.30; 95\% CI 1.16-1.52), whereas blacks, Asians, and Hispanics had lower odds (OR 0.52; 95\% CI 0.50-0.54, $p<0.001$; OR 0.88 ; $95 \%$ CI $0.83-0.94, p<0.001$; and OR $0.93 ; 95 \%$ CI $0.89-0.98, p<0.001$ respectively).

Overall, one-year mortality was $24.9 \%$ and 90 -day mortality was $9.1 \%$. In the known causes of death, cardiovascular was the most common cause of death (25.7\%) followed by withdrawal of dialysis (7.7\%). Figure 4 shows the adjusted ORs for gender and race predicting 1-year mortality in incident hemodialysis patients. There was no difference in mortality between males and females who were on incident hemodialysis (OR 1.01; 95\% CI 1.001.02). As compared to Whites, adjusted 1-year mortality was lower in blacks, Hispanics, Asians, and Native Americans (OR 0.67; 95\% CI 0.66-0.68, $p<0.001$; OR 0.62; 95\% CI $0.61-0.63, p<0.001$; OR 0.55 ; 95\% CI $0.53-0.56, p<$ 0.001 ; and OR 0.62 ; $95 \%$ CI $0.58-0.66, p<0.001$ respectively).

\section{Discussion}

Despite improved health outcomes associated with AV fistulas, $80 \%$ of Americans initiate hemodialysis using a catheter, and that rate can be influenced by several factors [6]. Our study is one of the largest studies, which examines a national cohort of all incident ESRD patients over the span of 10 years, for disparities in vascular access across 5 different racial groups and effect of differences in gender within each of the racial groups; and further accounts for parameters of pre-dialysis health. The current study demonstrates that females and Hispanics had lower likelihoods of using AV access to start dialysis than males and whites, respectively, whereas both blacks and Asians were more likely than whites to initiate dialysis with AV access. Additionally, only Native American patients among the non-white races had higher odds of AV fistula vs. AV graft for dialysis initiation than did whites.

Historically, black race has been associated with lower likelihood of initiating dialysis with an AV fistula $[15,16]$.
10

Am J Nephrol 2018;48:4-14 DOI: $10.1159 / 000490624$
Shah/Leonard/Meganathan/Christianson/ Thakar 


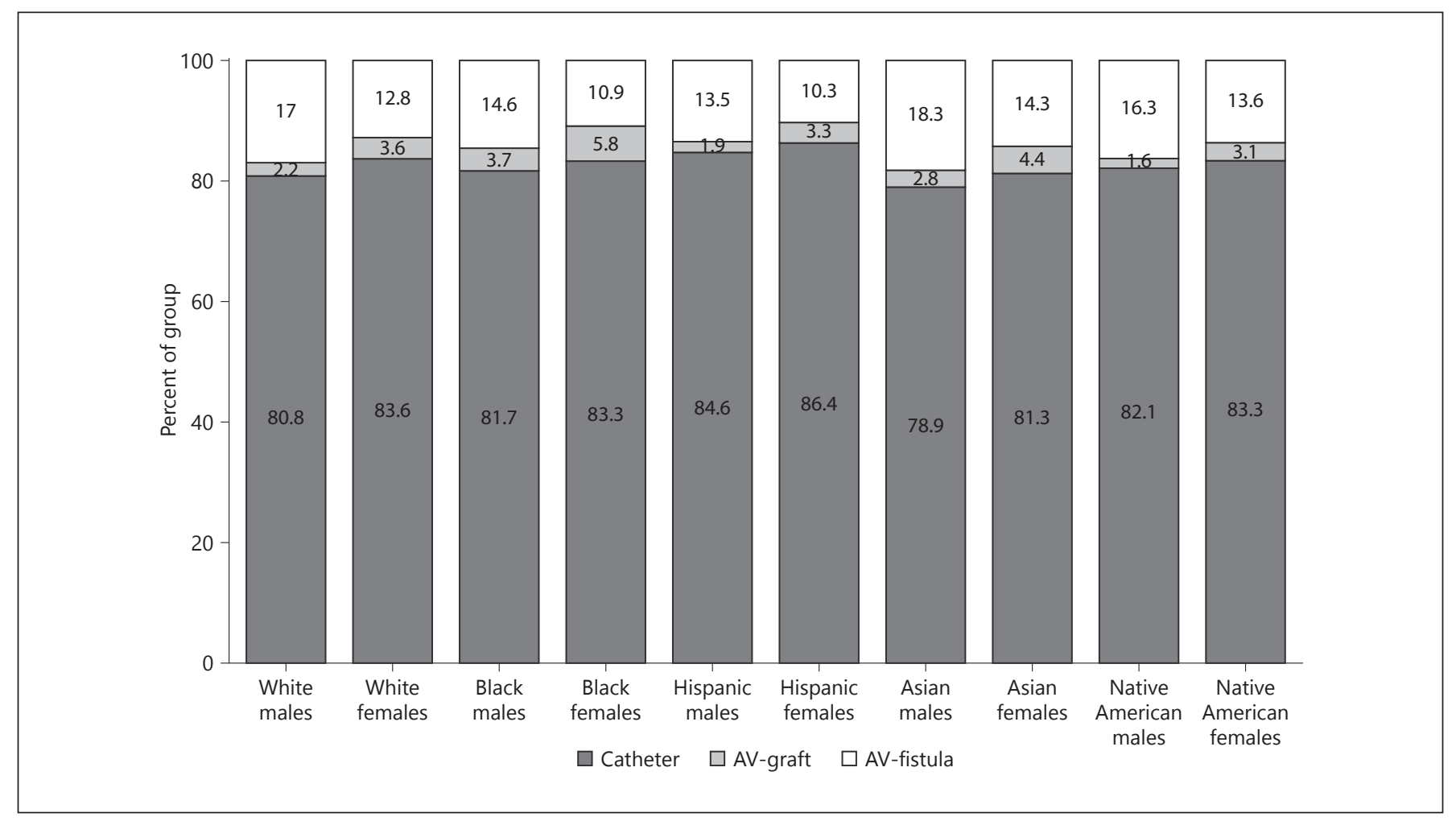

Fig. 2. Distribution of vascular access used for incident hemodialysis in the United States by race and gender. $p$ value $<0.001$ for difference in use of 3 access types across 5 races. $p$ value $<0.001$ for difference in use of 3 access types between males and females. $p$ value $<0.001$ for difference in use of 3 access types across 10 groups by race and gender. AV, arteriovenous.
Zarkowsky et al. [13] reported that white patients had a $22 \%$ greater chance than did black patients of initiating hemodialysis with an AV fistula versus combined groups of AV graft and catheter. Our study modeled the use of permanent AV access (AV fistula or AV graft) compared to catheter or others; and found that blacks had 8\% higher adjusted odds of using permanent access compared to whites. The likelihood of AV access was higher in blacks in spite of them receiving less pre-dialysis nephrology care and lacking insurance. Since white patients were older and had higher frequency of congestive heart failure, it is plausible that they initiated dialysis more frequently with a catheter. Furthermore, black patients had $48 \%$ lower odds of using AV fistula versus AV graft. Even though black patients were younger, our findings of higher AV graft use could be attributed to their higher rates of atherosclerotic heart disease and obesity, which is associated with failure to maturation of fistula, and compromised vascular anatomy impeding the use of native vessels for fistulae $[17,18]$.

Unlike the observations in black patients, compared to whites, Hispanics were $14 \%$ less likely to use AV access versus CVC; and were also less likely to use AV fistula versus AV graft for hemodialysis initiation. Furthermore, Hispanic patients of both gender had the lowest use of AV access. Prior studies have reported higher rates of diabetes mellitus, less pre-ESRD nephrology care, and lower levels of serum albumin at ESRD initiation in Hispanic patients, and these are important markers of preESRD quality of care [19-21] and our findings confirm this result. Arce et al. [16] showed that Hispanics are less likely to use AV access for first outpatient hemodialysis compared with non-Hispanics, and attribute this to variation in the access to pre dialysis nephrology care. Our study findings were similar; however, the disparity persisted even after adjusting for pre-dialysis health status including nephrology care, suggesting the influence of biological or other factors. We speculate that factors such as rate of progression of CKD or the timing of dialysis initiation in Hispanics may lead to suboptimal time necessary for planning and maturation of $\mathrm{AV}$ access prior to initial hemodialysis [22, 23].

Interestingly, Asian patients of both genders had the highest incidence of AV access use for hemodialysis ini- 
Fig. 3. Associations between race and gender and use of AV access for the initiation of hemodialysis among patients with ESRD in United States. Adjusted and unadjusted odds ratios for probability of (a) AV access use versus CVC to initiate hemodialysis, and (b) AV fistula use versus AV graft use to initiate hemodialysis. a Compared to white patients, the ORs, fully adjusted, and 95\% CIs for AV access in incident ESRD patients by race: blacks, 1.08 (1.07-1.10); Hispanics, 0.89 (0.87-0.90); Asians, 1.11 (1.07-1.14); and Native Americans, 1.04 (0.98-1.10). Compared to male patients, the ORs, fully adjusted, and 95\% CI for females, 0.85 (0.84-0.86). b Compared to white patients, the ORs, fully adjusted, and 95\% CIs for AV fistula in incident ESRD patients by race: blacks, $0.52(0.50-0.54)$; Hispanics, 0.93 (0.89-0.98); Asians, 0.88 (0.83-0.94); and Native Americans, 1.30 (1.12-1.52). Compared to male patients, the OR, fully adjusted, and 95\% CI for female patients, 0.45 (0.44-0.47). AV, arteriovenous; $\mathrm{CVC}$, central venous catheter.

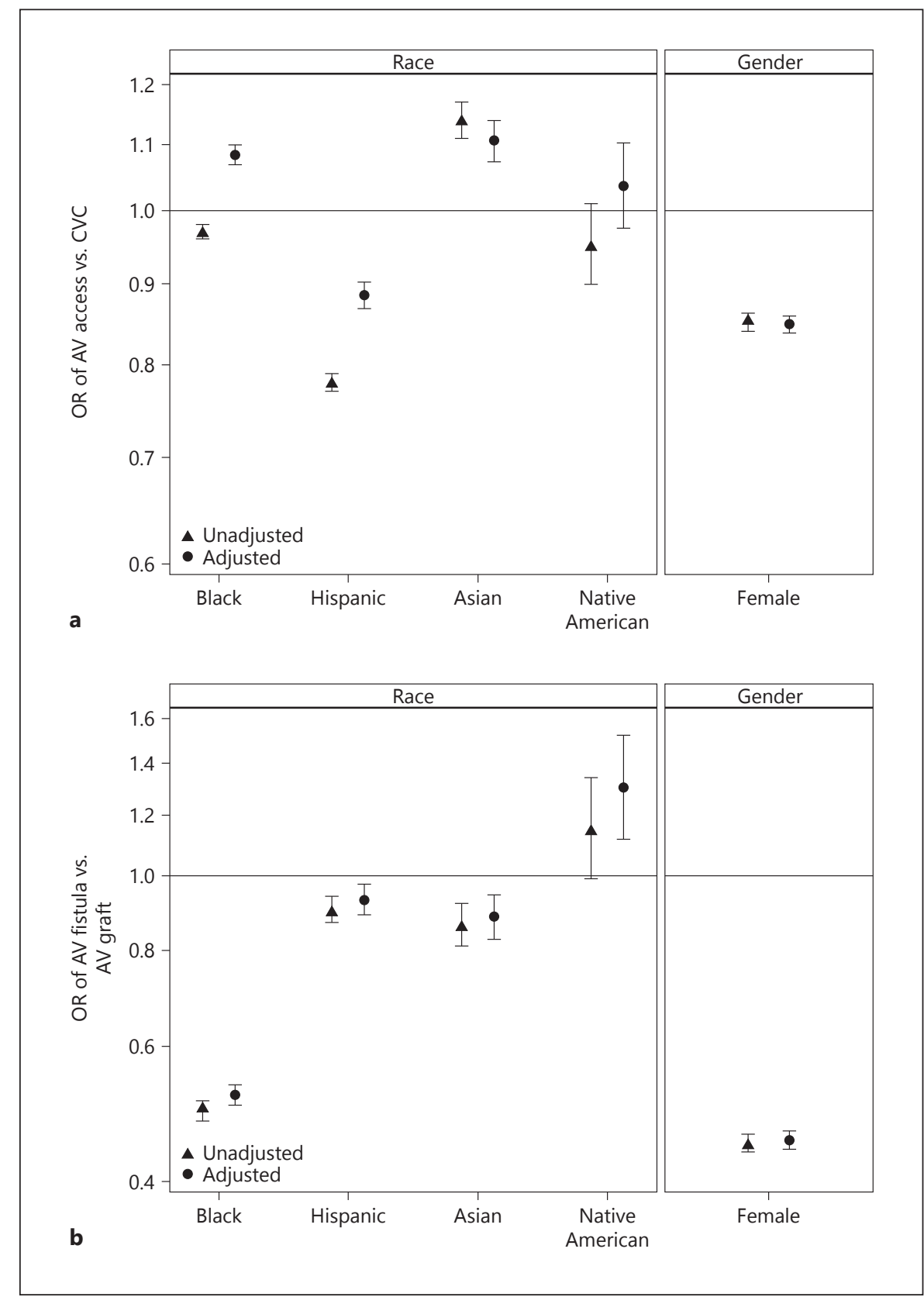

tiation. Frankenfield evaluated a national random sample of hemodialysis patients $(n=8,853)$ in the United States and reported that a higher percentage (36\%) of prevalent Asian patients were dialyzed with AV fistula than white patients (32\%) or black patients (26\%). However, they did not study the differences in the incident vascular access among Asian ESRD patients by gender [24]. In our study, despite lower adjusted odds of AV fistula versus AV graft in Asians as compared to whites, Asians patients had 11\% higher odds of AV access versus CVC. Biological differ- ences leading to lower tendency of thrombosis in Asians may contribute to them having a higher prevalence of $\mathrm{AV}$ access [25].

Gender-related differences continue to persist in the use of vascular access use for hemodialysis initiation. Markell et al. [26] analyzed 187,555 patients from USRDS; and reported that the rate of AV fistula use was $30 \%$ lower in women compared to men. Our study showed similar findings; and women, relative to men, had $15 \%$ adjusted lower odds of AV access versus CVC.
12
Shah/Leonard/Meganathan/Christianson/ Thakar 
Fig. 4. Associations between race and gender and 1 year mortality following initiation of hemodialysis among patients with ESRD. Adjusted and unadjusted OR for probability of 1-year mortality. Compared to white patients, the odds ratios, fully adjusted, and 95\% CIs in incident ESRD patients by race: blacks, 0.67 (0.66-0.68); Hispanics, 0.62 (0.61-0.63); Asians, 0.55 (0.53-0.56); and Native Americans, 0.62 (0.58-0.66). Compared to male patients, the OR, fully adjusted, and 95\% CI for female patients, 1.01 (1.00-1.02).

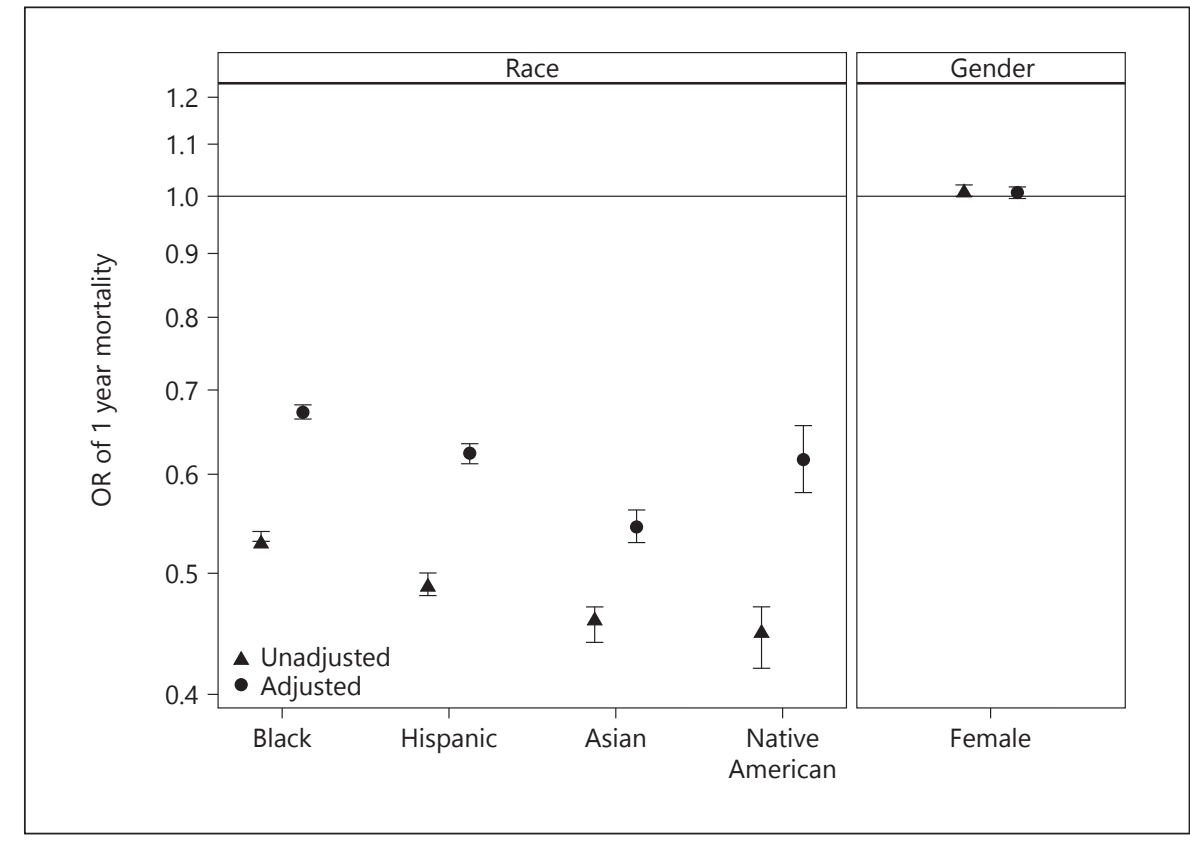

While many factors like no pre-dialysis nephrology care, lack of insurance, poor functional status, and comorbidities could contribute to the choice of vascular access among ESRD women, gender differences persisted even after accounting for all the significant factors for predialysis health. Surgical technique issues, referral patterns, or transition to care may be additional contributing factors. Women have smaller vessel diameters, and fistulas are less likely to mature in women than in men [27]. Additionally, the severity of CKD may be recognized later in women due to lower muscle mass, resulting in a delayed referral to a nephrologist. Moreover, patient preferences could play a role, with a higher preference among females for use of CVC due to "no sticks" with large needles, and no bleeding after dialysis, or cosmetic reasons [28]. This finding with marked gender disparity suggests some aspect of bias, either on the part of the physician or the patient.

The limitations of our study include the observational design, which precludes the determination of causality. Additionally, variability in the quality and completeness of the data recorded on form 2728 is associated with its inherent limitations for utilizing USRDS data. Yet, this represents one of the largest national cohorts of ESRD patients examining the type of vascular access, and gender and racial disparities. Other unmeasured variables such as educational level or household income may influence the associations, but these were not available in form 2728 for our analysis. However, we adjusted for pre-dialysis health including comorbidities, functional status, nursing home history, employment status and insurance, which may impact the choice of vascular access. The study also could not account for unmeasured confounders outside of the registry; however, by examining a large sample over a 10 -year period would potentially minimize any qualitative differences.

In conclusion, our study demonstrates significant disparities in vascular access use in hemodialysis patients in the United States, despite the translation of practice guidelines for hemodialysis vascular access into national clinical performance measures. The sociocultural underpinnings of these disparities deserve investigation aimed at understanding why certain populations do not receive the well-known benefits of this multidisciplinary approach to the care of patients with ESRD with regards to AV access placement. To eliminate these disparities, crafting successful solutions requires quality improvement strategies to improve the prevalence of AV access with a focus on selected races and genders; and physician and patient education about their practice patterns and possible biases.

\section{Disclosure Statement}

All the authors have no disclosures and competing interests. The results presented in this paper have not been published previously in whole or part, except in abstract format. The data reported here have been supplied by the US Renal Data System (USRDS). 
The interpretation and reporting of these data are the responsibility of the authors and in no way should be seen as official policy or interpretation of the US government.

\section{Funding Source}

S.S. is supported by a Junior Pilot Faculty Award from University of Cincinnati's Department of Internal Medicine.

\section{Author Contribution}

S.S. initiated the study, designed the study, and wrote the initial manuscript. A.C.L. contributed to the study design, analyzed and interpreted the data, and performed manuscript review. K.M. analyzed and interpreted the data; and reviewed the manuscript. A.C. contributed to the study figures, and manuscript review C.V.T. assisted S.S. with study design and implementation, revision of the manuscript and did the final approval of the manuscript. All authors reviewed the manuscript.

\section{References}

1 Volkova N, McClellan W, Klein M, Flanders D, Kleinbaum D, Soucie JM, Presley R: Neighborhood poverty and racial differences in ESRD incidence. J Am Soc Nephrol 2008; 19:356-364.

2 U.S. Renal Data System: Researcher's Guide to the USRDS Database. National Institutes of Health, National Institute of Diabetes and Digestive and Kidney Diseases, Bethesda, 2015.

3 Hecking M, Bieber BA, Ethier J, KautzkyWiller A, Sunder-Plassmann G, Saemann MD, Ramirez SP, Gillespie BW, Pisoni RL, Robinson BM, Port FK: Sex-specific differences in hemodialysis prevalence and practices and the male-to-female mortality rate: the Dialysis Outcomes and Practice Patterns Study (DOPPS). PLoS Med 2014;11: e1001750.

4 Kalantar-Zadeh K, Block G, Humphreys MH, Kopple JD: Reverse epidemiology of cardiovascular risk factors in maintenance dialysis patients. Kidney Int 2003;63:793-808.

5 Agodoa L, Eggers P: Racial and ethnic disparities in end-stage kidney failure-survival paradoxes in African-Americans. Semin Dial 2007;20:577-585.

6 United States Renal Data System: USRDS Annual Data Report: Epidemiology of Kidney Disease in the United States. National Institutes of Health National Institute of Diabetes and Digestive and Kidney Disease, Bethesda, 2016.

7 E. BJSBC: U.S. Department of Commerce. Economics and Statistics Administration. U.S. Census Bureau. The Asian Population: 2000. Census 2000 Brief February 2002.

8 Stevenson KB, Hannah EL, Lowder CA, Adcox MJ, Davidson RL, Mallea MC, Narasimhan N, Wagnild JP: Epidemiology of hemodialysis vascular access infections from longitudinal infection surveillance data: predicting the impact of NKF-DOQI clinical practice guidelines for vascular access. Am J Kidney Dis 2002;39:549-555.

9 Clinical practice guidelines for vascular access. Am J Kidney Dis 2006;48(suppl 1):S248 S273.

10 Lok CE, Mokrzycki MH: Prevention and management of catheter-related infection in hemodialysis patients. Kidney Int 2011;79: 587-598.

11 Lok CE, Foley R: Vascular access morbidity and mortality: trends of the last decade. Clin J Am Soc Nephrol 2013;8:1213-1219.

12 Pisoni RL, Young EW, Dykstra DM, Greenwood RN, Hecking E, Gillespie B, Wolfe RA, Goodkin DA, Held PJ: Vascular access use in Europe and the United States: results from the DOPPS. Kidney Int 2002;61:305316.

13 Zarkowsky DS, Arhuidese IJ, Hicks CW, Canner JK, Qazi U, Obeid T, Schneider E, Abularrage CJ, Freischlag JA, Malas MB: Racial/ethnic disparities associated with initial hemodialysis access. JAMA Surg 2015; 150:529-536

14 Astor BC, Eustace JA, Powe NR, Klag MJ, Sadler JH, Fink NE, Coresh J: Timing of nephrologist referral and arteriovenous access use: the CHOICE Study. Am J Kidney Dis 2001;38:494-501.

15 Trivedi PS, Lind KE, Ray CE, Rochon PJ, Ryu RK: Race and Sex Disparities in Outcomes of Dialysis Access Maintenance Interventions. J Vasc Interv Radiol 2018;29:476-481.e1.

16 Arce CM, Mitani AA, Goldstein BA, Winkelmayer WC: Hispanic ethnicity and vascular access use in patients initiating hemodialysis in the United States. Clin J Am Soc Nephrol 2012;7:289-296.

17 Reddan D, Klassen P, Frankenfield DL, Szczech L, Schwab S, Coladonato J, Rocco M, Lowrie EG, Owen WF Jr: National profile of practice patterns for hemodialysis vascular access in the United States. J Am Soc Nephrol 2002;13:2117-2124.

18 Lok CE, Allon M, Moist L, Oliver MJ, Shah $\mathrm{H}$, Zimmerman D: Risk equation determining unsuccessful cannulation events and failure to maturation in arteriovenous fistulas (REDUCE FTM I). J Am Soc Nephrol 2006; 17:3204-3212.

19 Nee R, Yuan CM, Hurst FP, Jindal RM, Agodoa LY, Abbott KC: Impact of poverty and race on pre-end-stage renal disease care among dialysis patients in the United States. Clin Kidney J 2017;10:55-61.
20 Fischer MJ, Go AS, Lora CM, Ackerson L, Cohan J, Kusek JW, Mercado A, Ojo A, Ricardo AC, Rosen LK, Tao K, Xie D, Feldman HI, Lash JP: CKD in Hispanics: Baseline characteristics from the CRIC (Chronic Renal Insufficiency Cohort) and Hispanic-CRIC Studies. Am J Kidney Dis 2011;58:214-227.

21 Murthy BV, Molony DA, Stack AG: Survival advantage of Hispanic patients initiating dialysis in the United States is modified by race. J Am Soc Nephrol 2005;16:782-790.

22 Derose SF, Rutkowski MP, Crooks PW, Shi JM, Wang JQ, Kalantar-Zadeh K, Kovesdy CP, Levin NW, Jacobsen SJ: Racial differences in estimated GFR decline, ESRD, and mortality in an integrated health system. Am J Kidney Dis 2013;62:236-244.

23 Kausz AT, Obrador GT, Arora P, Ruthazer R, Levey AS, Pereira BJ: Late initiation of dialysis among women and ethnic minorities in the United States. J Am Soc Nephrol 2000; 11:2351-2357.

24 Frankenfield DL, Ramirez SP, McClellan WM, Frederick PR, Rocco MV: Differences in intermediate outcomes for Asian and nonAsian adult hemodialysis patients in the United States. Kidney Int 2003;64:623-631.

25 White RH, Keenan CR: Effects of race and ethnicity on the incidence of venous thromboembolism. Thromb Res 2009;123(suppl 4): S11-S17.

26 Markell M, Brar A, Stefanov DG, Salifu MO: Gender disparity in fistula use at initiation of hemodialysis varies markedly across ESRD networks-Analysis of USRDS data. Hemodial Int 2017;22:168-175.

27 Frankenfield DL, Roman SH, Rocco MV, Bedinger MR, McClellan WM: Disparity in outcomes for adult Native American hemodialysis patients? Findings from the ESRD Clinical Performance Measures Project, 1996 to 1999. Kidney Int 2004;65:1426-1434.

28 Pisoni RL, Zepel L, Port FK, Robinson BM: Trends in US Vascular Access Use, Patient Preferences, and Related Practices: an Update From the US DOPPS Practice Monitor With International Comparisons. Am J Kidney Dis 2015;65:905-915. 